Supporting Information (revision jp0602427)

\title{
Structures of Tetrafluorocyclopropene, Hexafluorocyclobutene, Octafluorocyclopentene and Related Perfluoroalkene Radical Anions Revealed by ESR Spectroscopic and Computational Studies
}

\author{
Masaru Shiotani* \\ Department of Applied Chemistry, Graduate School of Engineering, \\ Hiroshima University, Higashi-Hiroshima 739-8527, Japan \\ Petter Persson and Sten Lunell \\ Department of Quantum Chemistry, Uppsala University, S-751 20 Uppsala, Sweden \\ Anders Lund \\ Department of Physics and Measurement Technology, Linköping University, S-581 83 Linköping, \\ Sweden \\ Ffrancon Williams \\ Department of Chemistry, University of Tennessee, Knoxville, Tennessee 37996-1600
}

3 Supporting Information

Title running head: Structure of perfluoroalkene radical anions

*Corresponding author: mshiota@hiroshima-u.ac.jp 
Supporting Information 1. Geometrical parameters computed for $c-\mathrm{C}_{3} \mathrm{~F}_{4}$ and $c-\mathrm{C}_{3} \mathrm{~F}_{4}{ }^{-}$. ${ }^{-}$

\begin{tabular}{|c|c|c|c|c|c|c|c|c|}
\hline & $\begin{array}{l}c \mathrm{C}_{3} \mathrm{~F}_{4} \\
\operatorname{Exp}^{\mathrm{b})},\end{array}$ & $c \mathrm{C}_{3} \mathrm{~F}_{4}$ & $c \mathrm{C}_{3} \mathrm{~F}_{4}$ & $c \mathrm{C}_{3} \mathrm{~F}_{4}$ & $c-\mathrm{C}_{3} \mathrm{~F}_{4}^{-}$ & $c-\mathrm{C}_{3} \mathrm{~F}_{4}^{-}$ & $c-\mathrm{C}_{3} \mathrm{~F}_{4}^{-}$ & $c-\mathrm{C}_{3} \mathrm{~F}_{4}^{-}$ \\
\hline $\begin{array}{c}\text { State } \\
\text { (symmetry) }\end{array}$ & & ${ }^{1} A\left(C_{2}\right)$ & ${ }^{1} A\left(C_{2}\right)$ & ${ }^{1} A\left(C_{2}\right)$ & ${ }^{2} A\left(C_{2}\right)$ & ${ }^{2} A\left(C_{2}\right)$ & ${ }^{2} A\left(C_{2}\right)$ & $\begin{array}{c}{ }^{2} A_{1} \\
\left(C_{2 \mathrm{v}}\right)\end{array}$ \\
\hline $\begin{array}{l}\text { Method/ } \\
\text { Basis set }\end{array}$ & & $\begin{array}{l}\text { B3LYP/ } \\
6-311+ \\
\text { G(d,p) }\end{array}$ & $\begin{array}{l}\text { MP2/6- } \\
311+\mathrm{G}( \\
\mathrm{d}, \mathrm{p})\end{array}$ & $\begin{array}{l}\mathrm{UHF} / 6- \\
311+\mathrm{G}( \\
\mathrm{d}, \mathrm{p})\end{array}$ & $\begin{array}{c}\text { B3LYP/6- } \\
311+G(d, \\
\text { p) }\end{array}$ & $\begin{array}{c}\mathrm{MP} 2 / 6- \\
311+\mathrm{G}( \\
\mathrm{d}, \mathrm{p})\end{array}$ & $\begin{array}{c}\mathrm{UHF} / 6-31 \\
1+\mathrm{G}(\mathrm{d}, \mathrm{p})\end{array}$ & $\begin{array}{c}\text { B3LYP/ } \\
6-311+ \\
\text { G(d,p) }\end{array}$ \\
\hline$l_{\mathrm{C}=\mathrm{C}}$ & $\begin{array}{l}1.296 \\
1.307 \\
\end{array}$ & 1.3046 & 1.3192 & 1.2818 & 1.3833 & 1.3907 & 1.3857 & 1.3034 \\
\hline$l_{\mathrm{C}-\mathrm{C}}$ & $\begin{array}{l}1.453 \\
1.461 \\
\end{array}$ & 1.4623 & 1.4660 & 1.4451 & 1.4425 & 1.4470 & 1.4399 & 1.4404 \\
\hline$l_{=\mathrm{C}-\mathrm{F}}$ & $\begin{array}{l}1.312 \\
1.315 \\
\end{array}$ & 1.3077 & 1.3056 & 1.2816 & 1.4111 & 1.3994 & 1.3718 & 1.3797 \\
\hline$l_{-\mathrm{C}-\mathrm{F}}$ & $\begin{array}{l}1.370 \\
1.361 \\
\end{array}$ & 1.3638 & 1.3573 & 1.3315 & 1.4131 & 1.4021 & 1.3697 & 1.3889 \\
\hline$<\mathrm{C}=\mathrm{C}-\mathrm{C}$ & $\begin{array}{l}63.5 \\
63.4 \\
\end{array}$ & 63.5 & 63.3 & 63.7 & 61.3 & 61.3 & 61.2 & 63.1 \\
\hline$<\mathrm{C}-\mathrm{C}-\mathrm{C}$ & & 53.0 & 53.5 & 52.7 & 57.3 & 57.4 & 57.5 & 53.8 \\
\hline$<\mathrm{C}=\mathrm{CF}$ & $\begin{array}{l}150.4 \\
149.9 \\
\end{array}$ & 150.1 & 150.1 & 150.2 & 131.3 & 131.4 & 130.3 & 145.2 \\
\hline$<\mathrm{CC}(=) \mathrm{F}$ & & 146.4 & 146.6 & 146.2 & 133.7 & 134.3 & 131.8 & 151.7 \\
\hline$<\mathrm{C}(=) \mathrm{CF}$ & & 122.7 & 122.6 & 122.7 & $\begin{array}{l}119.4 \\
128.3 \\
\end{array}$ & $\begin{array}{r}119.2 \\
128.2 \\
\end{array}$ & $\begin{array}{l}119.1, \\
127.8 \\
\end{array}$ & 123.5 \\
\hline$<\mathrm{FCF}$ & $\begin{array}{l}104.1 \\
105.4\end{array}$ & 105.7 & 105.9 & 105.8 & 101.5 & 101.8 & 102.3 & 103.6 \\
\hline $\begin{array}{c}\text { dihedral } \\
\text { angle } \\
\text { between } \\
\mathrm{C}-\mathrm{F} \text { bond } \\
\text { and } \\
\mathrm{C}=\mathrm{C}-\mathrm{C} \\
\text { plane }\end{array}$ & & 0.0 & 0.0 & 0.0 & 20.6 & 20.2 & 21.4 & 0.00 \\
\hline
\end{tabular}

a) Bond length in $\AA$, bond angle in angle and energy in a.u.

b) Ref. 47. Upper: $156 \mathrm{~K}$ by X-ray crystallography; Lower: In gas phase by a combined analysis of electron-diffraction data, rotation constants derived from the microwave spectrum, and dipolar coupling constants derived from the liquid crystal ${ }^{19} \mathrm{~F}$ NMR spectrum. 
Supporting Information 2. Geometrical parameters computed for $c-\mathrm{C}_{4} \mathrm{~F}_{6}$ and $c-\mathrm{C}_{4} \mathrm{~F}_{6}{ }^{-}$. ${ }^{\text {) }}$

\begin{tabular}{|c|c|c|c|c|c|c|c|}
\hline & $\begin{array}{l}c-\mathrm{C}_{4} \mathrm{~F}_{6} \\
\exp ^{\mathrm{b}, \mathrm{c})}\end{array}$ & $c-\mathrm{C}_{4} \mathrm{~F}_{6}$ & $c-\mathrm{C}_{4} \mathrm{~F}_{6}$ & $c-\mathrm{C}_{4} \mathrm{~F}_{6}$ & $c-\mathrm{C}_{4} \mathrm{~F}_{6}^{-}$ & $c-\mathrm{C}_{4} \mathrm{~F}_{6}^{-}$ & $c-\mathrm{C}_{4} \mathrm{~F}_{6}^{-}$ \\
\hline $\begin{array}{l}\text { State } \\
\text { (symmetry) }\end{array}$ & $\begin{array}{c}D_{2 \mathrm{~h}} \\
\text { (coplanar) }\end{array}$ & ${ }^{1} \mathrm{~A}^{\prime}\left(C_{\mathrm{s}}\right)$ & ${ }^{1} \mathrm{~A}^{\prime}\left(C_{\mathrm{s}}\right)$ & ${ }^{1} \mathrm{~A}^{\prime}\left(C_{\mathrm{s}}\right)$ & $\begin{array}{l}{ }^{2} \mathrm{~A}\left(C_{1}\right) \\
\text { twisted }\end{array}$ & $\begin{array}{l}{ }^{2} \mathrm{~A}\left(C_{1}\right) \\
\text { twisted }\end{array}$ & $\begin{array}{l}{ }^{2} \mathrm{~A}\left(C_{1}\right) \\
\text { twisted }\end{array}$ \\
\hline $\begin{array}{l}\text { Geometry } \\
\text { (method } \\
\text { /basis sets) }\end{array}$ & & $\begin{array}{c}\text { B3LYP/6-311 } \\
+\mathrm{G}(\mathrm{d}, \mathrm{p})\end{array}$ & $\begin{array}{c}\mathrm{MP} 2 / 6-311+ \\
\mathrm{G}(\mathrm{d}, \mathrm{p})\end{array}$ & $\begin{array}{c}\mathrm{UHF} / 6-311+ \\
\mathrm{G}(\mathrm{d}, \mathrm{p})\end{array}$ & $\begin{array}{c}\text { B3LYP/6-311 } \\
+\mathrm{G}(\mathrm{d}, \mathrm{p})\end{array}$ & $\begin{array}{c}\text { MP2/6-311+ } \\
\mathrm{G}(\mathrm{d}, \mathrm{p})\end{array}$ & $\begin{array}{c}\text { UHF/6-311+ } \\
\text { G(d,ffp) }\end{array}$ \\
\hline$l_{\mathrm{C}=\mathrm{C}}$ & $\begin{array}{r}1.342 \pm \\
\left.0.002^{\mathrm{b}}\right) \\
1.3336^{\mathrm{c})}\end{array}$ & 1.3376 & 1.3489 & 1.3145 & 1.4170 & 1.4230 & 1.4169 \\
\hline$l_{=\mathrm{C}-\mathrm{C}}$ & $\begin{array}{l}1.508^{ \pm} \\
0.003^{\mathrm{b})} \\
1.4786^{\mathrm{c}}\end{array}$ & 1.5020 & 1.4986 & 1.4941 & 1.4748 & 1.4762 & 1.4793 \\
\hline$l_{\mathrm{C}-\mathrm{C}}$ & $\begin{array}{l}1.595^{\mathrm{b})} \\
1.5526^{\mathrm{c})}\end{array}$ & 1.5781 & 1.5669 & 1.5512 & 1.5519 & 1.5433 & 1.5347 \\
\hline$l_{=\mathrm{C}-\mathrm{F}}$ & $\begin{array}{l}1.319 \pm \\
0.012^{\mathrm{b})}\end{array}$ & 1.3108 & 1.3087 & 1.2878 & 1.3947 & 1.3866 & 1.3661 \\
\hline$l_{\text {-C-F }}$ & $\begin{array}{l}1.336 \pm \\
0.006^{\text {b) }}\end{array}$ & 1.3526 & 1.3483 & 1.3214 & 1.3903 & 1.3829 & 1.3516 \\
\hline$<\mathrm{C}=\mathrm{C}-\mathrm{C}$ & $\begin{array}{c}94.8 \pm 0.3^{b)} \\
94.32^{\mathrm{c})}\end{array}$ & 94.6 & 94.2 & 94.5 & 91.7 & 91.0 & 91.0 \\
\hline$<=\mathrm{C}-\mathrm{C}-\mathrm{C}$ & $\begin{array}{c}85.2^{\text {b) }} \\
85.72^{c)}\end{array}$ & 85.4 & 85.8 & 85.5 & 86.6 & 86.4 & 86.6 \\
\hline$<\mathrm{C}=\mathrm{CF}$ & $\begin{array}{c}133.6 \pm 0.3 \\
\text { b) }\end{array}$ & 134.9 & 135.2 & 135.3 & 125.8 & 118.7 & 125.2 \\
\hline$<\mathrm{FCF}$ & & 107.5 & 107.8 & 107.6 & 103.2 & 103.6 & 103.9 \\
\hline $\begin{array}{c}\mathrm{C}-\mathrm{C}=\mathrm{C}-\mathrm{C} \\
\text { torsional } \\
\text { angle }\end{array}$ & 0.00 & 0.00 & 0.00 & 0.00 & 14.4 & 16.9 & 16.6 \\
\hline $\begin{array}{c}\mathrm{F}-\mathrm{C}=\mathrm{C}-\mathrm{F} \\
\text { torsional } \\
\text { angle }\end{array}$ & 0 & 0 & 0 & 0 & 77.6 & 76.8 & 80.2 \\
\hline $\begin{array}{c}\text { dihedral } \\
\text { angle } \\
\text { between } \\
\mathrm{C}-\mathrm{F} \text { bond } \\
\text { and } \\
\mathrm{C}=\mathrm{C}-\mathrm{C} \\
\text { plane }\end{array}$ & 0 & 0 & 0 & 0 & 20.8 & 21.3 & 21.8 \\
\hline
\end{tabular}


Supporting Information 3: Electron energies and oscillator strengths of $\mathrm{C}_{2} \mathrm{~F}_{4}{ }^{-}, c-\mathrm{C}_{3} \mathrm{~F}_{4}{ }^{-}, c-\mathrm{C}_{4} \mathrm{~F}_{6}{ }_{6}$ and $c-\mathrm{C}_{5} \mathrm{~F}_{8}{ }^{-}$anions computed by TD-DFT methods. The data related to the first five excited states are given.

\begin{tabular}{|c|c|c|c|}
\hline $\begin{array}{l}\text { Perfluoroalkene } \\
\text { radical anion }\end{array}$ & $\begin{array}{l}\text { Excitation } \\
\text { energies/nm }\end{array}$ & $\begin{array}{c}\text { Oscillator strengths: } \\
\mathrm{f}\end{array}$ & $\begin{array}{l}\text { Main electron transition: } \\
\text { state to state (contribution) }\end{array}$ \\
\hline $\mathrm{C}_{2} \mathrm{~F}_{4}^{-}$ & $\begin{array}{ll}1: & 473.34 \\
\text { 2: } & 343.92 \\
\text { 3: } & 338.46 \\
\text { 4. } & 321.88 \\
\text { 5. } & 302.07\end{array}$ & $\begin{array}{l}0.0680 \\
0.1027 \\
0.0534 \\
0.0000 \\
0.1024 \\
\end{array}$ & $24 \mathrm{~B}->25 \mathrm{~B}(0.93341)$ \\
\hline$c-\mathrm{C}_{3} \mathrm{~F}_{4}^{-}$ & $\begin{array}{ll}1: & 443.07 \\
2: & 337.17 \\
3: & 321.53 \\
4: & 304.45 \\
5: & 277.49\end{array}$ & $\begin{array}{l}0.0124 \\
0.1129 \\
0.0005 \\
0.0510 \\
0.0585\end{array}$ & $27 \mathrm{~B}->28 \mathrm{~B}(0.95857)$ \\
\hline$c-\mathrm{C}_{4} \mathrm{~F}_{6}^{-}$ & $\begin{array}{ll}1: & 404.34 \\
2: & 365.42 \\
3: & 333.24 \\
4: & 323.90 \\
5: & 302.66\end{array}$ & $\begin{array}{l}0.0058 \\
0.0010 \\
0.1238 \\
0.0549 \\
0.0127 \\
\end{array}$ & 39B -> 40B (0.76544) \\
\hline$c-\mathrm{C}_{5} \mathrm{~F}_{8}^{-}$ & $\begin{array}{ll}1: & 436.02 \\
2: & 399.57 \\
3: & 332.89 \\
4: & 331.70 \\
5: & 325.12\end{array}$ & $\begin{array}{l}0.0080 \\
0.0027 \\
0.0068 \\
0.0495 \\
0.1012\end{array}$ & $52 \mathrm{~A}->54 \mathrm{~A}(0.81912)$ \\
\hline
\end{tabular}

Time-dependent (TD) B3LYP/6-311+G (d,p)// UHF/ 6-311+G (d,p) methods were used. 